(body), QoL, satisfaction, anxiety, depression and patient global assessment. Patient satisfaction was most commonly used $(n=10)$ as single domain with a wide variation in wording of questions and answering categories. The absolute cut-off value was the most common type of response, with large variety in value and timing of follow-up. Table 1 shows one definition for each combination of domains.

Conclusion: Our review shows that definitions for poor response to TKR are ambiguous. Our findings stress the need for an unambiguous definition of poor response to draw conclusions about the prevalence of poorresponders to TKR across hospitals and countries, and to identify patients at risk.

Abstract THU0461 -Table 1. The table shows one definition for each combination of domains

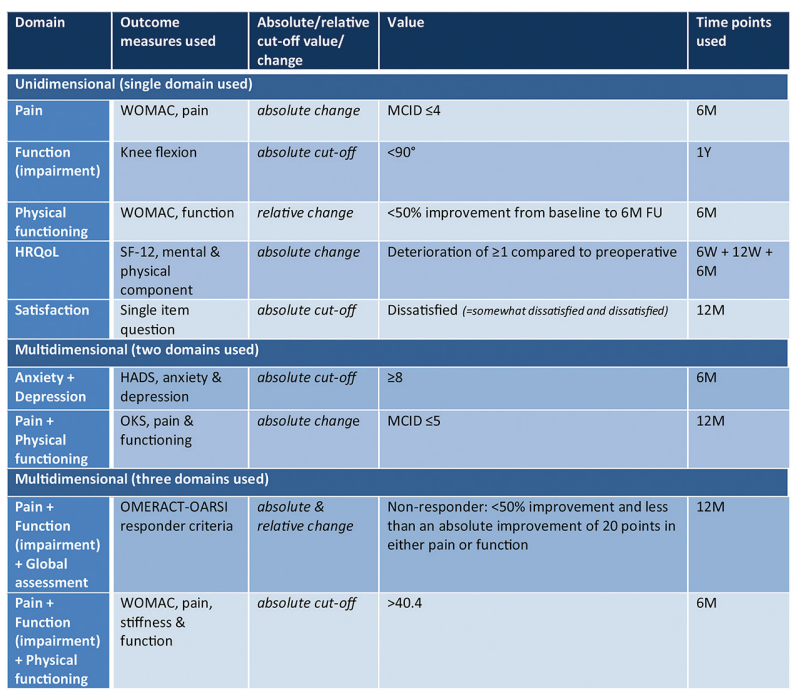

Disclosure of Interests: : None declared

DOI: 10.1136/annrheumdis-2019-eular.7983

\section{THU0462 OUTCOME PREDICTION FOR TREATMENT OF KNEE OSTEOARTHRITIS WITH A TOTAL KNEE ARTHROPLASTY. DEVELOPMENT AND VALIDATION OF A PREDICTION MODEL FOR PAIN AND FUNCTIONAL OUTCOME USING THE DUTCH ARTHROPLASTY REGISTER (LROI) DATA}

J.J. Tolk ${ }^{1}$, J.H. Waarsing ${ }^{2}$, R.P.A. Janssen ${ }^{1}$, L.N. van Steenbergen ${ }^{3}$, S.M. A. Bierma-Zeinstra ${ }^{2,4}$, Max Reijman ${ }^{4}{ }^{1}$ Máxima Medical Centre Eindhoven, Department of orthopaedic surgery and trauma, Eindhoven, Netherlands; ${ }^{2}$ Erasmus MC, University Medical Center Rotterdam, Department of general practice, Rotterdam, Netherlands; ${ }^{3}$ Dutch Arthroplasty Registry, 's Hertogenbosch, Netherlands; ${ }^{4}$ Erasmus MC, University Medical Center Rotterdam, Department of orthopaedic surgery, Rotterdam, Netherlands

Background: One of the main determinants of treatment satisfaction after total knee arthroplasty (TKA) is the fulfilment of preoperative expectations. For optimal expectation management it is useful to be able accurately predict the treatment result. Multiple patient factors that are obtained for registration in the Dutch Arthroplasty Registry (LROI) are associated with the treatment result. Therefore, these factors can potentially be utilised to estimate the most likely outcome on pain and functional outcome for an individual patient

Objectives: The aim of the present study was to create and validate models that predict residual symptoms on 10 specific outcome parameters at 12-month follow-up for patients undergoing primary TKA for knee osteoarthritis.

Methods: Data was extracted from the LROI on TKA patients who had pre- and postoperative PROMs registered in the LROI registry. Multiple logistic regression analyses were performed to construct predictive algorithms for satisfaction, treatment success, and residual symptoms concerning pain in rest and during activity, sit-to-stand movement, stair negotiation, walking, performance of activities of daily living, kneeling and squatting. Models were developed for men and women separately. We assessed predictive performance by examining measures of calibration and discrimination.

Results: Data of 7071 patients could be included for data analysis Residual complaints on kneeling ( $\left.\$ 72 \% / \sigma^{x} 59 \%\right)$ and squatting ( $\$ 71 \% /$ ${ }^{\prime} 56 \%$ ) were reported most frequently, and least residual complaints were scored for walking $\left(\$ 16 \% / \sigma^{x} 12 \%\right)$ and pain in rest ( $\left.18 \% / 0^{x} 14 \%\right)$. The predictive algorithms for residual symptoms concerning sit-to-stand movement, stair negotiation, walking, activities of daily living and treatment success showed acceptable discriminative values (AUC $0.68-0.74$ ). The prediction models for residual complaints regarding kneeling, squatting, pain and satisfaction showed the least favourable results (AUC 0.58 0.64). The calibration curves showed adequate calibration for most of the models.

Conclusion: A considerable proportion of patients has residual complaints after TKA. The present study showed that demographic and PROMs data collected for the LROI registry, can be used to predict the chance for residual symptoms after TKA. The predictive models that have been developed can be useful for individual expectation management in patients planned for TKA for knee osteoarthritis.

Acknowledgement: This study was supported by a grant from the Van Rens Foundation, and conducted with data of the Dutch Arthroplasty Registry.

Disclosure of Interests: None declared

DOI: 10.1136/annrheumdis-2019-eular.1654

\section{THU0463 EXPECTATIONS OF TREATMENT RESULT OF KNEE OSTEOARTHRITIS PATIENTS TREATED WITH A TOTAL KNEE ARTHROPLASTY. THE INFLUENCE OF DEMOGRAPHIC FACTORS, PAIN, PERSONALITY TRAITS, PHYSICAL AND PSYCHOLOGICAL STATUS}

J.J. Tolk ${ }^{1}$, R.P.A. Janssen ${ }^{1}$, T.M. Haanstra ${ }^{2}$, M. (Marieke) C. van der Steen ${ }^{2}$, S.M. A. Bierma-Zeinstra ${ }^{3,4}$, Max Reijman ${ }^{4} .^{1}$ Máxima Medical Center, Department of Orthopedic Surgery and Trauma, Eindhoven, Netherlands; ${ }^{2}$ Radboud University Medical Center, Department of Orthopaedic Surgery, Nijmegen, Netherlands ${ }^{3}$ Erasmus MC, University Medical Center Rotterdam, Department of General Practice, Rotterdam, Netherlands; ${ }^{4}$ Erasmus MC, University Medical Center Rotterdam, Department of Orthopedic Surgery, Rotterdam, Netherlands

Background: Unrealistic preoperative expectations have a strong influence on the outcome after total knee arthroplasty (TKA). More insight into determinants of the level of expectations is useful in identifying patients at risk for unrealistic expectations. This information can be used in optimizing pre-operative expectation management.

Objectives: The aim of the current study was to analyze to what extent pre-operative outcome expectations of TKA patients are determined by psychological factors, demographic factors, pain, physical function and general health status.

Methods: A cross-sectional analysis of 204 patients with symptomatic and radiographic knee OA, scheduled for primary TKA was conducted. Outcome expectations were measured using the Hospital for Special Surgery knee replacement expectations survey. Independent variables included were age, sex, body mass index and patient reported outcome measures for pain, physical function, quality of life, anxiety, depression, catastrophizing, optimism and pessimism. Multiple linear regression analyses were used to evaluate associations between these variables and pre-operative outcome expectations

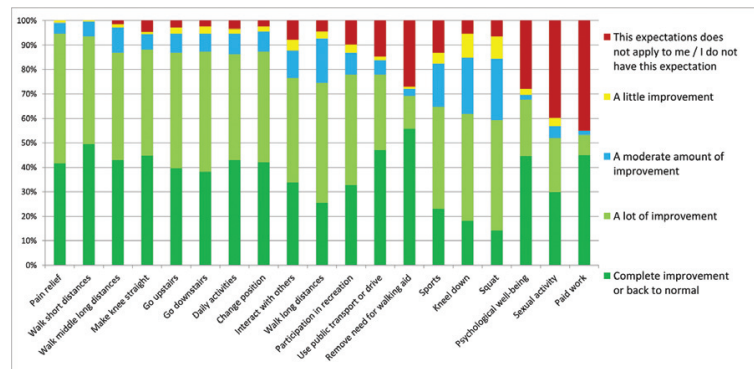

Abstract THU0463 - Figure 1

Results: The mean overall survey score on the HSS-KRES was 70.9 (SD 17.9). Distribution of expectation scores is shown in Figure 1. 
Highest expectations were scored for pain relief and improvement of the ability to walk of short and medium distances. Patients had the lowest expectations for improvement in kneeling, squatting, psychological wellbeing sexual activity and the ability to have paid work.

Female sex, higher age, higher depression score and duration of complaints $>50$ months showed to be significant predictors of lower expectations for the treatment outcome after TKA. Baseline pain and function scores were not related to the level of pre-operative expectations.

Conclusion: In conclusion young, male patients with a short duration of complaints might be at risk of having too high expectations of the treatment result. On the contrary patients with depressive symptoms are more likely to have low expectations, with a potential negative influence on their treatment result. The present study aids in identifying patients at risk for having either too high or too low expectations. This knowledge can be utilized in individualized expectation management interventions.

Acknowledgement: We would like to thank $\mathrm{C}$. van Doesburg and $\mathrm{H}$. Kox for their support in data collection and study procedures.

Disclosure of Interests: None declared

DOI: 10.1136/annrheumdis-2019-eular.5123

\section{THU0464 PHASE 2 CLINICAL TRIAL OF THE GI SAFETY OF A HYDROGEN SULFIDE-RELEASING ANTI- INFLAMMATORY DRUG (ATB-346)}

John Wallace ${ }^{1}$, Andre Buret ${ }^{1}$, Peter Nagy ${ }^{2}$, Marcelo Muscara ${ }^{3}$, Gilberto de Nucci ${ }^{4}$. ${ }^{1}$ University of Calgary, Calgary, Canada; ${ }^{2}$ National Institute of Oncology, Budapest, Hungary; ${ }^{3}$ University of Sao Paulo, Sao Paulo, Brazil; ${ }^{4}$ University of Campinas, Campinas, Brazil

Background: Hydrogen sulfide $\left(\mathrm{H}_{2} \mathrm{~S}\right)$ is a naturally occurring gaseous mediator produced by intestinal bacteria and various eukaryotic cells. $\mathrm{H}_{2} \mathrm{~S}$ exerts anti-inflammatory, pro-resolution and cytoprotective effects in vivo. ATB-346 is an $\mathrm{H}_{2} \mathrm{~S}$-releasing derivative of naproxen, which in animals was shown to produce negligible gastrointestinal (GI) damage and bleeding. In human studies, ATB-346 was found to be much more potent and long-lasting than naproxen. A phase 2 open-label efficacy study demonstrated that ATB-346 (250 mg daily) significantly reduced pain in patients with osteoarthritis of the knee, and markedly suppressed cyclooxygenase (COX) activity. The aim of the present study was to determine if ATB346 would induce less gastroduodenal ulceration than standard dose naproxen.

Objectives: To determine if healthy subjects taking ATB-346 for 14 days would develop significantly less gastroduodenal ulcers $(\geq 3 \mathrm{~mm}$ diameter with depth) than subjects taking an equi-effective dose of naproxen

Methods: This was a double-blind, active control, endoscopic study. 244 healthy volunteers completed the study. Upper Gl endoscopy was performed prior to and on day 14 after commencing treatment with naproxen (550 mg twice daily) or ATB-346 (250 mg) once daily in the morning and placebo once daily in the evening. Whole blood thromboxane synthesis was measured on days 0,7 and 14 . Plasma $\mathrm{H}_{2} \mathrm{~S}$ levels were also measured.

Results: 53 subjects taking naproxen (42.2\%) developed at least one ulcer, while only 3 subjects (2.5\%) treated with ATB-346 developed at least one ulcer $(\mathrm{p}<0.0001)$. The two drugs suppressed COX activity to the same extent $(>95 \%)$. Affected subjects in the naproxen group developed more ulcers (an average of 4 per subject) than in the ATB-346 group (an average of 1.3), and there was a much greater incidence of larger ulcers $(\geq 5 \mathrm{~mm}$ diameter) in the naproxen group than in the ATB346 group (125 vs 0 , respectively). The incidence of gastro-esophageal reflux, abdominal pain and nausea was lower with ATB-346 than with naproxen. Plasma $\mathrm{H}_{2} \mathrm{~S}$ levels were significantly elevated (by $50 \%$; $p<0.001)$ in the ATB-346 group.

Conclusion: Consistent with the pre-clinical studies, this phase 2 clinical trial demonstrated a dramatic reduction of upper Gl ulcer formation in subjects treated with equi-effective doses of ATB-346 versus naproxen. The COX inhibition observed in this trial was consistent with a previous phase $2 \mathrm{~A}$ trial that demonstrated significant pain relief with ATB-346 in patients with osteoarthritis of the knee. ATB-346 appears to be an effective and much safer alternative to existing NSAIDs.

Disclosure of Interests: : John Wallace: None declared, Andre Buret Shareholder of: Antibe Therapeutics, Peter Nagy: None declared, Marcelo Muscara Grant/research support from: Antibe Therapeutics, Gilberto de Nucci Shareholder of: Antibe Therapeutics, Employee of: BioLab Brasil DOI: 10.1136/annrheumdis-2019-eular.568

\section{THU0464B IS THERE AN ASSOCIATION BETWEEN METABOLIC SYNDROME AND SEVERITY OF HAND OSTEOARTHRITIS? RESULTS FROM A NATIONWIDE STUDY}

Margarida Cruz ${ }^{1}$, Alexandre Sepriano ${ }^{2}$, Sara Dias ${ }^{3}$, Ana Maria Rodrigues ${ }^{4}$ Helena Canhão ${ }^{3}$, Nélia Gouveia ${ }^{2}$, Mónica Eusébio ${ }^{5}$, Sofia Ramiro ${ }^{6}$, Jaime Branco ${ }^{7}{ }^{1}$ Nova Medical School - Lisbon, Lisbon, Portugal; ${ }^{2}$ Faculdade de Ciências Médicas, CEDOC, Lisboa, Portugal, ${ }^{3}$ Faculdade de Ciências Médicas, CEDOC, EpiDoc, Lisboa, Portugal; ${ }^{4}$ Faculdade de Ciências Médicas, Lisboa, Portugal; ${ }^{5}$ Sociedade Portuguesa de Reumatologia, Lisboa, Portugal; ${ }^{6}$ Leiden University Medical Center, Leiden, Netherlands; ${ }^{7}$ Nova Medical School, CEDOC, Lisboa, Portugal

Background: Hand osteoarthritis ( $\mathrm{HOA})$ is a highly prevalent rheumatic disease that predominates in females and causes pain, joint deformities and loss of functional capacity. Overweight and metabolic syndrome have been previously suggested to associate with the severity of HOA, but clarity on these associations is yet to be achieved.

Objectives: To test the possible association between body mass index (BMI) and other individual components of metabolic syndrome with severity of $\mathrm{HOA}$ in females from a nationwide epidemiological study.

Methods: EpiReumaPt was a three-stage national health survey where, in the first phase, 10,661 adult participants were randomly selected and interviewed using a structured face-to-face questionnaire that included screening for rheumatic diseases, such as HOA. In the second phase, positive screenings for $\geq 1$ rheumatic complaint plus $20 \%$ of the negative screenings were invited for an assessment by rheumatologists. Finally, 3 rheumatologists revised all the information and defined the final diagnosis by consensus. Female patients with a final clinical diagnosis of primary HOA were included in this analysis. Hand functional status as assed by the Cochin questionnaire was the outcome of interest. The explanatory variables of interest were: BMI evaluated as a categorical variable (Normal: 18-24.99; overweight: 25-29.99; obesity: $\geq 30$ ), diabetes mellitus, hypertension and hypercholesterolemia (all self-reported and as binary variables: yes/no). The possible associations between BMI and the individual components of the metabolic syndrome with the Cochin score were tested in a multivariable linear regression model. Only significant variables $(p<0,05)$ were kept in the final model. Potential confounders of the associations of interest and the outcome were defined a priori on clinical grounds and included age and symptoms of depression (HADS score).

Results: Out of the 3,877 participants evaluated by Rheumatologists, 473 women had primary HOA (national prevalence: $6.6 \%$ ). In this population, $40 \%$ were overweight and $29 \%$ were obese. Ninety-three $(20 \%)$ participants had diabetes, 261 (56\%) had hypertension and 261 (56\%) had hypercholesterolemia. In the multiple regression model, BMI and diabetes were found to significantly associate with HOA severity, whereas hypertension and hypercholesterolemia did not, thus not being selected in the final model (table).

Abstract THU0464 -Table 1. Association between individual components of the metabolic syndrome and HOA severity (Cochin score). Multivariable linear regression model

\begin{tabular}{lc} 
& HOA severity (Cochin score) \\
$\beta$ coefficient $(95 \% \mathrm{Cl})$ & $\mathrm{N}=408$ \\
\hline BMl (categorical) & $0.31(0.05 ; 0.57)$ \\
Diabetes (yes vs no) & $3.63(0.13 ; 7.13)$ \\
Age (years) & $0.13(-0.01 ; 0.27)$ \\
HADS score (continuous) & $0.90(0.59 ; 1.22)$ \\
\hline
\end{tabular}

Conclusion: In this study, higher BMI and the presence of diabetes mellitus associated with a worse functional capacity in women with primary HOA. These data add to the body of evidence suggesting a possible role of metabolic factors in the severity of HOA.

\section{REFERENCES:}

[1] Yusuf

E, Nelissen RG, loan-Facsinay A et al. Association between weight or body mass index and hand osteoarthritis: a systematic review. Ann Rheum Dis 2010. 69: 761-765

[2] Schett G, Kleyer A, Perricone $C$ et al. Diabetes is an independent predictor for severe osteoarthritis: results from a longitudinal cohort study. Diabetes Care 2013; 36: 403-409

Acknowledgement: The authors thank all the participants of EpiReumaPt survey, the interviewers and staff and the Rheumatologists that worked in the field Disclosure of Interests: Margarida Cruz: None declared, Alexandre Sepriano: None declared, Sara Dias: None declared, Ana Maria 